\title{
Kapazitive Gasphasendetektion in flüssigem Stickstoff
}

\author{
Christoph Kandlbinder ${ }^{1}$, Alice Fischerauer ${ }^{1}$, Mario Mösch ${ }^{1}$, Tobias Helling ${ }^{1}$, Gerhard Fischerauer ${ }^{1}$, \\ Martin Siegl ${ }^{2}$ \\ ${ }^{1}$ Lehrstuhl für Mess- und Regeltechnik sowie Zentrum für Energietechnik (ZET), Universität Bayreuth, \\ 95440 Bayreuth, Deutschland \\ ${ }^{2}$ Institut für Raumfahrtsysteme, Deutsches Zentrum für Luft- und Raumfahrt e.V. (DLR), \\ 28359 Bremen, Deutschland \\ +49 (0)921 55-7236, christoph.kandlbinder@uni-bayreuth.de
}

\section{Zusammenfassung}

Die Ober- und Hauptstufen von Trägerraketen werden häufig mit kryogenen Flüssigkeiten betrieben. Besonders für eine Zündung des Triebwerkes unter Schwerelosigkeit ist es wichtig, das Verhalten dieser kryogenen Flüssigkeiten zu beobachten. Dabei stellen Gasblasen, welche in die Treibstoffzuleitungen gelangen und das Zünden eines Triebwerks verhindern können, ein Risiko dar. Zur Messung einer entstehenden Gasphase und ihres Volumens haben wir ein kapazitives Messsystem für Zweiphasengemische realisiert, welches ohne Energieeintrag in die Flüssigkeit eine Füllstandsänderung unter tiefkalten Bedingungen detektieren kann. Dabei sind die Elektroden so angeordnet, dass Phasenänderungen am Rand, etwa eine an der Behälterseite stattfindende Verdampfung, erfasst werden können. Die charakteristischen Kapazitätsverläufe der unterschiedlichen Elektrodenpaarungen wurden experimentell bestimmt und einerseits mit Finite-ElementeSimulationen (Ansys) verglichen. Andererseits wurden Berechnungen zur elektrischen Flussdichte angestellt, welche die simulierten Kapazitätsverläufe mit theoretischen Aussagen ergänzen. Die experimentell ermittelten Ergebnisse spiegeln die Simulationsdaten dabei mit nur geringen Abweichungen wieder. Durch Messung der Teilkapazitäten und der Kapazitäten gegen Masse ließ sich sowohl eine hohe Empfindlichkeit auf eine Phasenänderung an den Wänden realisieren, als auch der tatsächliche Füllstand eindeutig zuordnen.

Keywords: Kapazitive Sensoren, Mehrelektrodensysteme, Zweiphasengemische, kryogene Flüssigkeiten

\section{Einleitung}

Für künftige Raumfahrzeuge, wie die geplante europäische Rakete ARIANE 6, spielt die Wiederzündbarkeit eine Schlüsselrolle für die Entwicklung der mit kryogenen Treibstoffen betriebenen Oberstufe. Dies würde das Wechseln des Orbits und damit neue Anwendungen für die Raumfahrt ermöglichen. Um die dabei auftretenden Fluid-Phänomene beobachten und gegebenenfalls beeinflussen zu können, ist es insbesondere bei teilgefüllten Tanks von enormer Bedeutung zu wissen, wo sich die flüssige Phase befindet und wie die Topologie der freien Oberfläche beschaffen ist. Da bei geringer Schwerkraft die Kapillarkräfte dominieren, bestimmen diese die Form und Position der freien Oberfläche. Zusätzlich können durch Schwappvorgänge, einer Veränderung der Stärke der Gravitation oder durch einen Wärmeeintrag Gasblasen entstehen. Gelangen diese in die Treibstoffzuleitungen, können die Turbopumpen beschädigt werden, wodurch eine Wiederzündung unmöglich wird. Um diese Phänomene zu untersuchen, sind Experimente mit geeigneten Messsystemen notwendig [1].

Um eine ungewollte Aufheizung der kryogenen Flüssigkeit zu verhindern, dürfen Messsysteme nur vernachlässigbar wenig Energie in die Flüssigkeit eintragen. Außerdem muss ein solches System unter tiefkalten Bedingungen funktionieren, während Aussagen über die örtliche Verteilung von Gasphasen getroffen werden sollen, das System aber dabei weder invasiv noch intrusiv sein sollte.

Derzeitige Messmethoden für kryogene Umgebungen verwenden optische, mikrowellenbasierte, resistive oder kapazitive Prinzipien. Jedoch können diese zu einer lokalen Aufheizung oder anderen störenden Effekten führen [2].

Kapazitive Sensoren besitzen ein hohes Potential, um die Oberflächenposition einer 
Flüssigphase zu bestimmen. Da ein kapazitives System eine Phasenänderung zwischen zwei Elektroden misst, gibt es weder eine Auswirkung auf die Bewegung, noch eine Erwärmung des Fluids. Die Elektroden können so in die Tankwand eingebaut und integriert werden, dass einerseits keine zusätzlichen Sensorstrukturen nötig sind und andererseits der Tank nicht geometrisch modifiziert werden muss.

\section{Kryostat}

Um das nachfolgend beschriebene Messsystem in kryogenen Flüssigkeiten testen zu können, wurde ein Kryostat als Testumgebung benutzt (Abb. 1).

Der innere Tank des Kryostaten kann dabei mit einem Druck von bis zu 4 bar beaufschlagt werden, wobei der Zwischenraum zwischen dem inneren und äußeren Tank mit flüssigem Stickstoff befüllt werden kann, um einen zusätzlichen Schutz vor einem Wärmeeintrag von außen zu bieten. Im inneren Tank ist ein Folienheizdraht befestigt, welcher zur Blasenerzeugung mittels lokalem Wärmeeintrag in den flüssigen Stickstoff dient.

Das Flüssig/Dampf-System im Kryostaten wird entweder auf der Siedelinie oder in einem leicht unterkühlten Zustand gehalten. Durch Änderung des Druckes ist es daher möglich, das Volumen von gasförmig vorliegendem Stickstoff zu kontrollieren. Steigt der Druck im Kryostaten, nimmt das Gasvolumen aufgrund von Kondensationsprozessen $a b$, da die Flüssigkeit weiter unterkühlt wird. Dies ist in einem Druck-Temperatur-( $p$-T-)Diagramm sichtbar (Abb. 2). Der Arbeitspunkt ist durch einen roten Kreis markiert, der sich auf der Siedelinie bzw. knapp unter dieser im gasförmigen Bereich befindet. Erhöht sich der Druck, wandert der Arbeitspunkt in vertikaler Richtung in den flüssigen Bereich (blauer Kreis). Somit kondensiert ein sich innerhalb der Flüssigkeit befindliches gasförmiges Volumen aus, und das Gas-zu-FlüssigVerhältnis innerhalb dieses Volumens lässt sich kontrollieren.

Der Folienheizdraht hingegen erlaubt das Erzeugen von Blasen, indem er den flüssigen Stickstoff lokal aufheizt, wodurch es je nach Heizleistung zu Blasen- oder Filmsieden kommen kann. Die so entstehenden Blasen steigen dann zur Oberfläche des flüssigen Stickstoffes auf. Werden sie durch eine dafür bestimmte Vorrichtung aufgefangen, verbinden sie sich und bilden ein Gasvolumen innerhalb des flüssigen Stickstoffes (LN2).

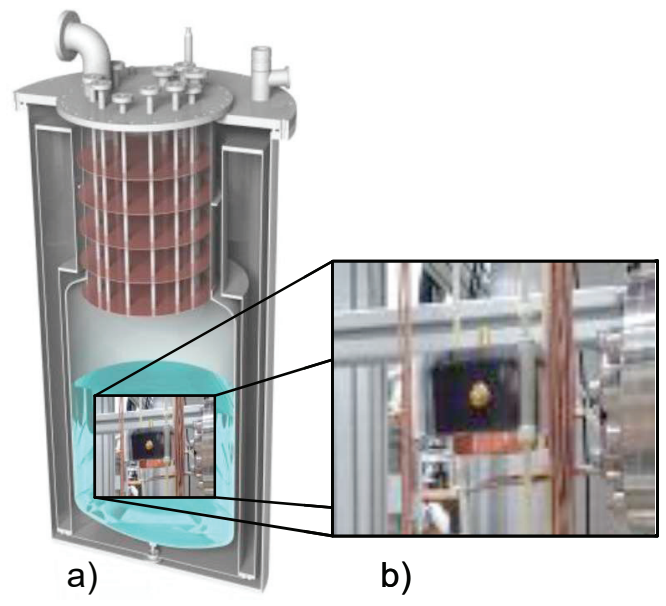

Abb. 1: Kryostat mit a) Gesamtaufbau und b) eingebautem innerem Zylinder.

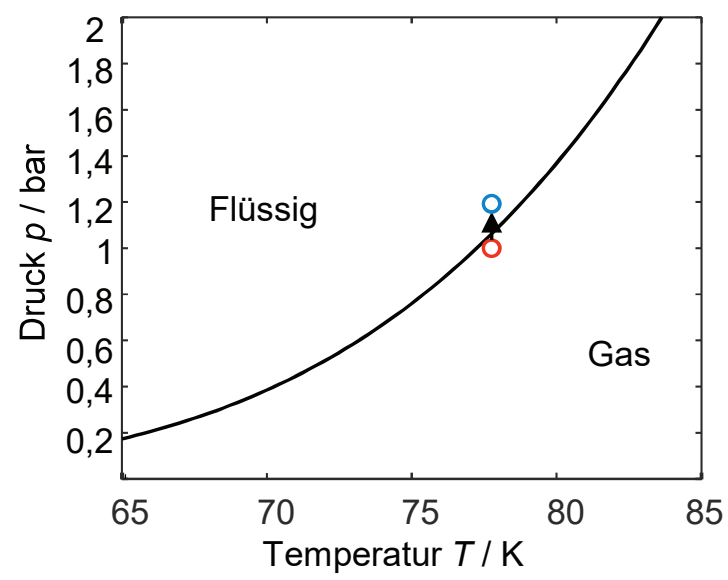

Abb. 2: Druck-Temperatur-(p-T-)Diagramm von flüssigem Stickstoff (LN2) [3].

\section{Messsystem}

Das aufgebaute Messsystem besteht aus einem nach unten geöffneten PolycarbonatZylinder, der vollständig in den mit LN2 gefüllten Kryostaten eingetaucht wird und aus dem Flüssigvolumen aufsteigende Gasblasen einfängt. Diese lokalen Phasenänderungen von flüssigem zu gasförmigem Stickstoff werden über die Kapazitäten zwischen verschiedenen Elektroden an der Zylinderoberfläche (Innenmantel und Deckel) gemessen. Dies ist möglich, weil sich die Permittivitäten von flüssigem und gasförmigem Stickstoff unterscheiden $\left(\varepsilon_{\mathrm{r}, \mathrm{LN} 2} \approx 1,43, \varepsilon_{\mathrm{r}, \mathrm{N} 2(\mathrm{~g})} \approx 1,0\right)$ [4].

Die Zylinder- und Elektrodengeometrie ist in Abb. 3 dargestellt. Die Kapazitäten werden dabei mit einem IC des Typs Acam PCap02AD gemessen. Dieses IC bestimmt die Kapazität durch die Messung der Entladezeit über einen definierten Vorwiderstand. Das IC kann dabei softwaretechnisch zwischen der Messung von Kapazitäten gegen Masse und der Messung von Teilkapazitäten zwischen zwei Elektroden umgeschaltet werden [5]. 


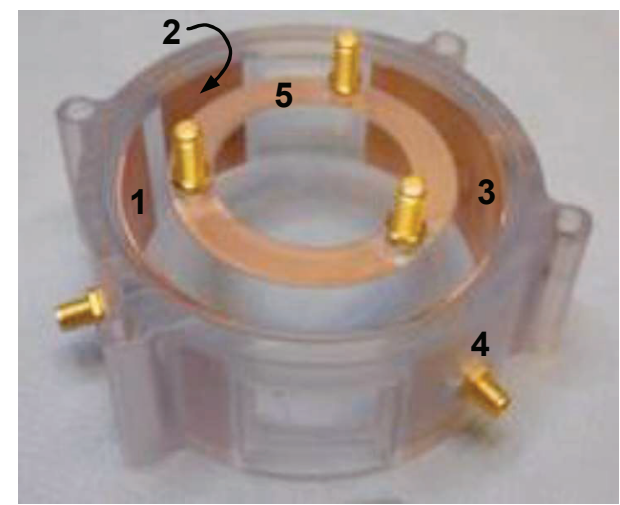

Abb. 3: Verwendeter Polycarbonat-Zylinder mit fünf Elektroden.

Im Experiment wurden Gasblasen im flüssigen Stickstoff definiert wie im Abschnitt zuvor beschrieben mit Hilfe eines Folienheizdrahts unter dem Zylinder erzeugt. Diese Blasen konnten sich in dem nach oben geschlossenen Zylinder ansammeln und zu einer expandierenden Gasphase vereinen. Mit einer Kamera, die von der Seite auf den Zylinder gerichtet war, wurde der relative Anteil der Gasphase im Zylinderinneren während der Kapazitätsmessung optisch überwacht.

\section{Modellierung und Simulation der Kapazitä- ten}

Um die gemessenen Ergebnisse zu validieren und zu interpretieren, wurden Finite-Elemente Simulationen mit dem Softwarewerkzeug Ansys durchgeführt. Die modellierte Geometrie entspricht dabei der realen Geometrie von Abb. 1b (Abb. 4).

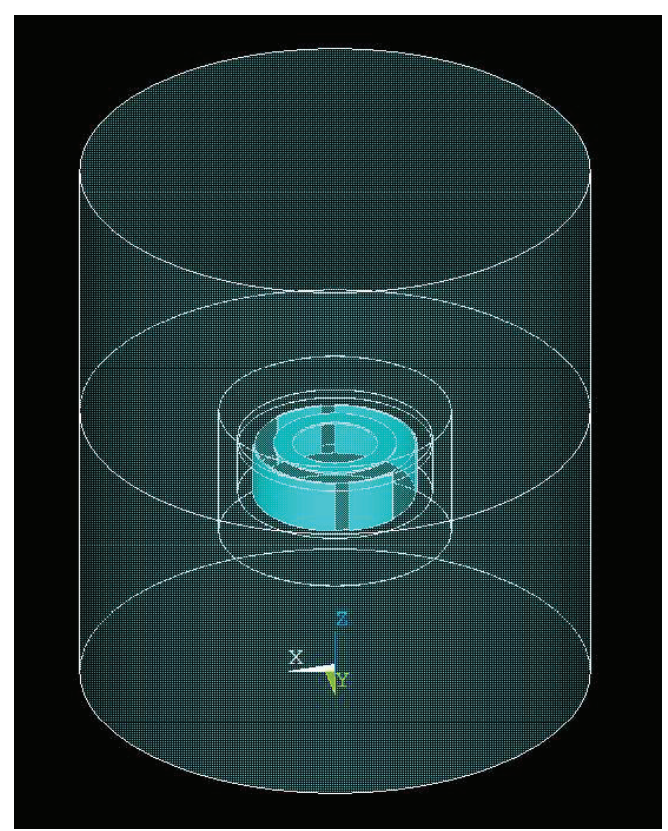

Abb. 4: In der Simulation verwendete Geometrie für den Zylinder mit hochaufgelöstem Elementnetz um den Zylinder herum.
Wenn Blasen innerhalb des LN2 aufsteigen, verbinden sie sich im Zylinderinnenraum, und der LN2-Füllstand $L$ in diesem Innenraum verändert sich. Die Simulationsergebnisse in Abb. 5 demonstrieren, wie sich dies auf ausgewählte Teilkapazitäten auswirkt. Dabei wurde mit $C_{i j}$ die Teilkapazität zwischen den Elekroden $i$ und $j$ bezeichnet (Nummerierung der Elektroden wie in Abb. 3).

Nach Abb. 5a hängt die Kapazität $C_{13}(L)$ bei niedrigen Füllständen $(L<20 \mathrm{~mm})$ quasi-linear von $L$ ab. Sie erreicht ein Maximum bei $L \approx 23 \mathrm{~mm}$ und sinkt für weiter steigende Füllstände dann wieder. Aus Gründen der Empfindlichkeit und aus Gründen der Eindeutigkeit lassen sich mit den Elektroden 1 und 3 folglich nur Füllstände unterhalb von ca. $20 \mathrm{~mm}$ messen.

Bei $C_{25}(L)$ ist es gerade umgekehrt (Abb. $5 b$ ). Diese Teilkapazität ändert sich für geringe Füllstände $(L<20 \mathrm{~mm})$ nur wenig. Bei hohen Füllständen $(L=20 \ldots 30 \mathrm{~mm})$ reagiert sie hingegen stark auf den Füllstand. Diese mit $L$ zunehmende Empfindlichkeit ist plausibel, da Elektrode 5 auf dem Zylinderdeckel sitzt und daher vom Füllstand umso mehr beeinflusst wird, je höher er ist.

Ähnlich, aber nicht völlig identisch liegen die Verhältnisse bei den Kapazitäten einzelner Elektroden gegen Masse (Abb. 6). Die Empfindlichkeit dieser Kapazitäten steigt für hohe Füllstände, ganz besonders wenn der Zylinder annähernd voll mit LN2 ist. Dabei sind
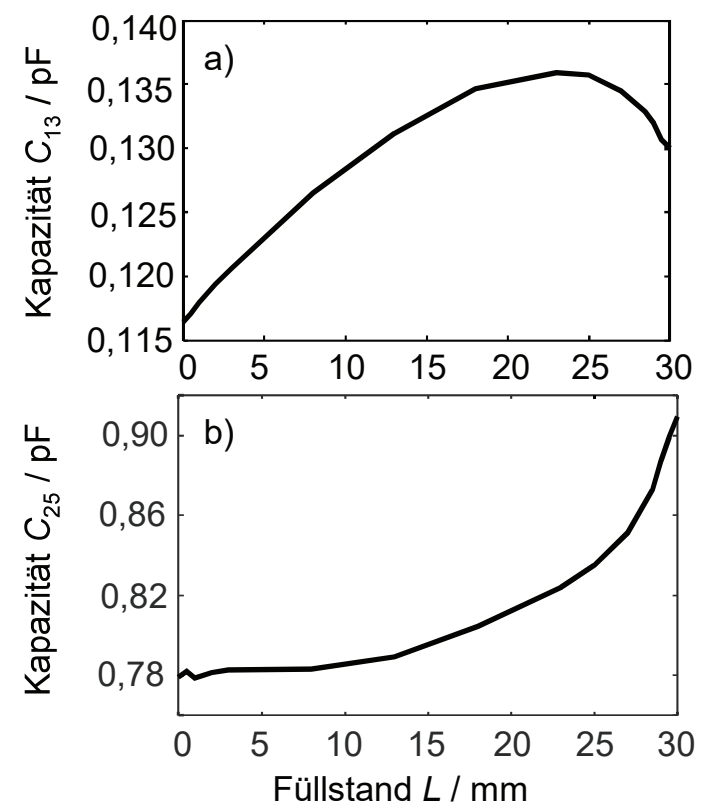

Abb. 5: Simulierte Teilkapazitäten unterschiedlicher Elektrodenpaare als Funktion des LN2-Füllstands. a) $\mathrm{C}_{13}$. b) $\mathrm{C}_{25}$. 

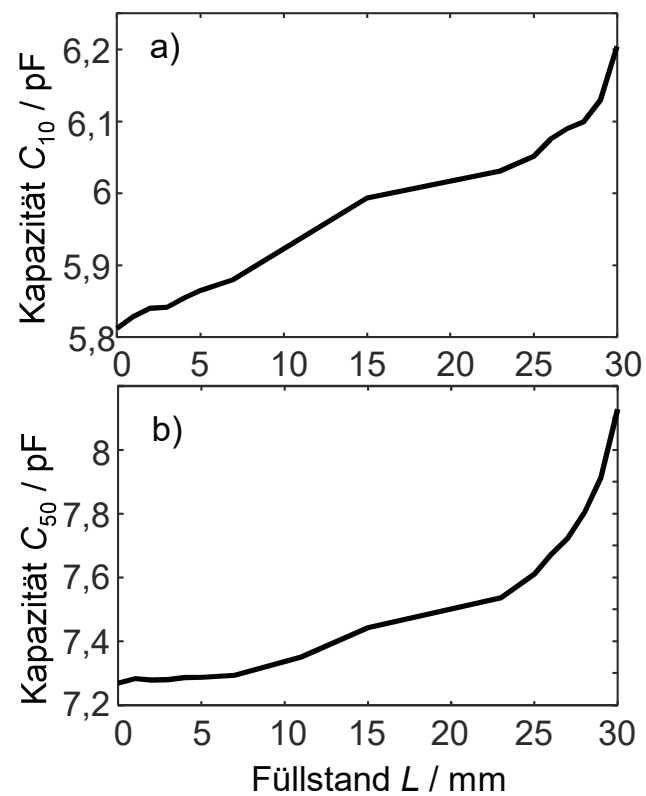

Abb. 6: Simulierte Kapazitäten ausgewählter Elektroden gegen Masse als Funktion des LN2-Füllstands. a) Elektrode 1. b) Elektrode 5.

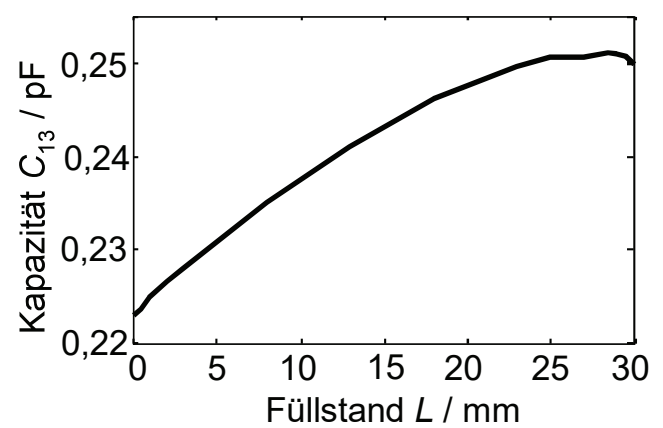

Abb. 7: Simulierte Teilkapazität $C_{13}$ für eine Massepotentialabweichung von $2 \%$ des aktiven Elektrodenpotentials.

die Änderungen der Kapazitäten gegen Masse über die gesamte Füllhöhe betrachtet um etwa eine Größenordnung größer als die Änderungen der entsprechenden Teilkapazitäten (einige $0,1 \mathrm{pF}$ gegenüber einigen $0,01 \mathrm{pF}$ ).

Offensichtlich eignen sich die Kapazitäten gegen Masse gut dafür, Gasphasenanteile in der Nähe der jeweils betrachteten Elektroden zu erfassen, da dort eine unschwer nachweisbare Kapazitätsänderung auftritt. Praktisch werden jedoch die Teilkapazitäten für die elektrische Kapazitätstomografie (ECT) benötigt, mit der Phasengrenzen im Volumen zwischen den Elektroden aufgedeckt werden können [6].

Die Messung dieser Teilkapazitäten wird im Feld durch nichtideale Masseverbindungen beeinflusst. Man vergleiche hierzu Abb. $5 a\left(C_{13}\right.$ mit perfekter Masse an den Elektroden 2, 4 und 5) mit Abb. 7 ( $C_{13}$ mit den Elektroden 2, 4 und 5 auf einem Potential, das $2 \%$ des Potentials der aktiven Elektroden entspricht).

\section{Feldstärkeverlauf}

Zur Plausibilisierung und Deutung der simulierten Kapazitätsverläufe wurde numerisch das elektrostatische Feld in der Umgebung des elektrodentragenden Zylinders bestimmt (FERechnungen mit Ansys). Dies werde hier am Beispiel der Potentialverteilung, die zur Messung der Teilkapazität $C_{13}(L)$ gehört, diskutiert. In Abb. 8 sind der Betrag und (in weiß) die Feldlinien der elektrischen Flussdichte $\vec{D}(\vec{r})$ für drei verschiedene LN2-Füllstände dargestellt.

a)

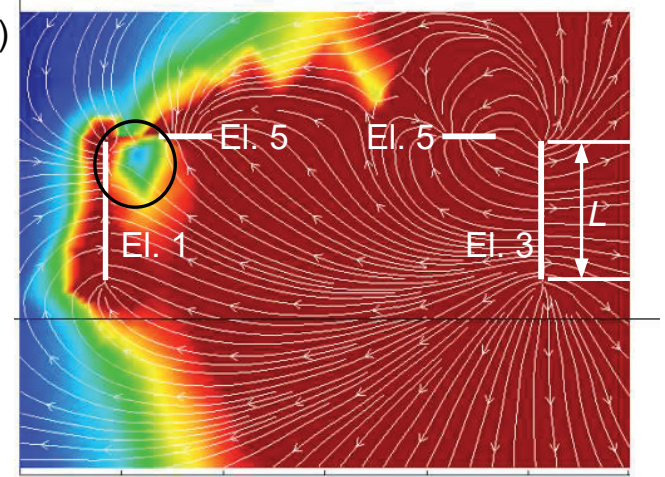

b)

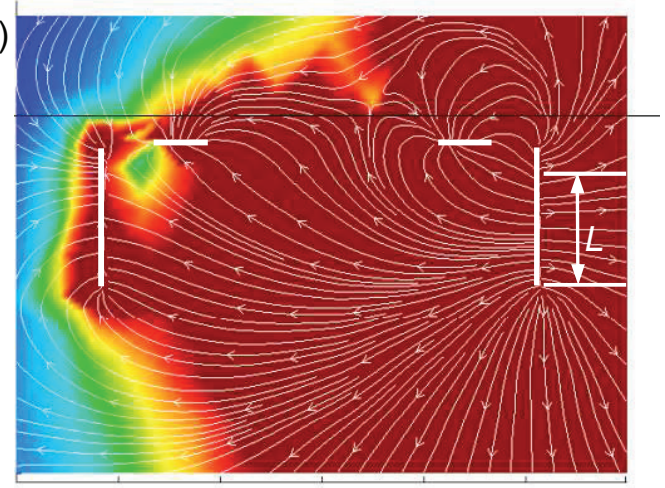

c)
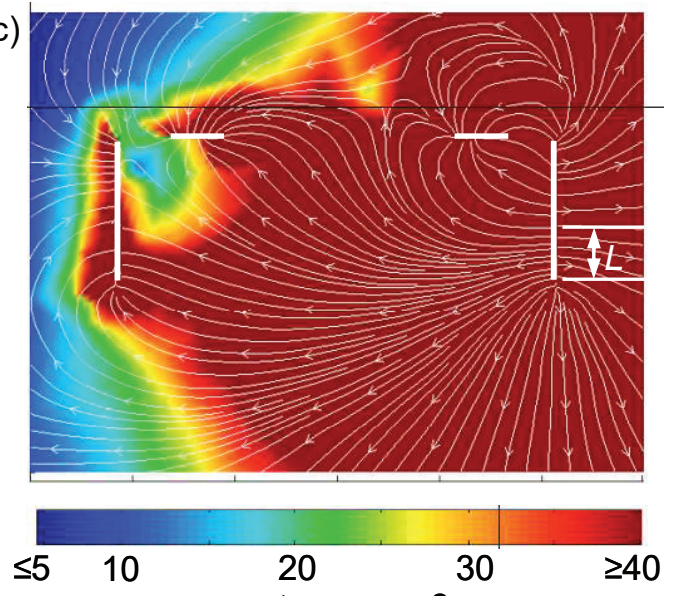

d)

$$
|\vec{D}| /\left(\mathrm{pC} / \mathrm{m}^{2}\right)
$$

Abb. 8: Betrag und Feldlinien der elektrischen Flussdichte bei Anregung der Wandelektrode 3 für verschiedene $L N 2-F u ̈ l l s t a ̈ n d e \quad L$ a) $L=30 \mathrm{~mm}$ (Zylinder voll mit LN2). b) $L=25 \mathrm{~mm}$. c) $L=10 \mathrm{~mm}$. d) Farbskala für die Betragsdarstellung. 
In Abwesenheit von Gaseinschlüssen (Abb. 8a) ist das Feld im Gebiet $\mathrm{G}$ zwischen den Elektroden 1 und 5 (schwarz umrandeter Bereich in Abb. 8a) schwächer als im unteren Teil der Elektrode 1 (man beachte die Farbskala in Abb. 8d). Die von der aktiven Elektrode 3 ausgehenden Feldlinien enden teils auf Elektrode 1, teils auf Elektrode 5.

Bei Ausbilden einer Gasphase oberhalb des LN2 wächst die Feldstärke im Gebiet $G$ an (Abb. 8b). Auf der geerdeten Elektrode 5 landen weniger Feldlinien als zuvor, weil die Elektrode an die Gasphase grenzt und das Feld sich im höher permittiven LN2 konzentriert. Da Elektrode 1 zum größten Teil an das LN2 grenzt, landen nun dort die nicht mehr zu Elektrode 5 führenden Feldlinien, und die Kapazität $C_{13}$ steigt gegenüber vorher an.

Wird allerdings die Dicke der Gasphasenschicht zu groß (Abb. 8c), schwächt sich auch das Feld im Gebiet $G$ wieder ab. Da nun auch Elektrode 1 größtenteils an die Gasphase grenzt, hat sie relativ zu Elektrode 5 keinen Vorteil mehr beim „Einfangen“ von Feldlinien. Folglich verringert sich $C_{13}$ ab diesem Punkt nur noch mit sinkendem LN2-Füllstand.

\section{Messungen und Ergebnisse}

Verschiedene Befüllungs- und Leerzyklen wurden experimentell durchgeführt. Immer wenn der LN2-Füllstand aufgrund von Kondensationsprozessen anstieg, wurden ausgewählte Kapazitäten gemessen und sowohl die Gas/flüssig-Phasengrenze, als auch die Aktivität der Blasen optisch über eine Kamera überwacht. Mithilfe dieser Kamera wurde jeweils der momentane Füllstand bestimmt.

Abbildung 9 zeigt den gemessenen LN2-Füllstand $L$ als Funktion der Zeit für ein typisches
Experiment. Hier wurden die Teilkapazitäten $C_{13}$ und $C_{25}$ gemessen. Aus innen wurde der Füllstand $L$ errechnet, indem die theoretisch ermittelten Kennlinien $C_{13}(L)$ und $C_{25}(L)$ aus Abb. 5a, b invertiert wurden.

Bei $\mathrm{t} \approx 450 \mathrm{~s}$ war der Zylinderinnenraum ganz mit gasförmigem Stickstoff befüllt (vollständig LN2-frei). Danach stieg die Gas/flüssig-Phasengrenze annähernd linear mit der Zeit an, bis der Raum bei $t \approx 450 \mathrm{~s}$ vollständig mit LN2 gefüllt war. Bei $t \approx 650 \mathrm{~s}$ wurde der Heizdraht angeschaltet. In der Folge stiegen Blasen auf und verdrängten den flüssigen Stickstoff im Zylinderinnenraum. Ab diesem Zeitpunkt sank daher der LN2-Füllstand wieder.

Wie erwartet, reagiert $C_{13}$ empfindlich auf das Einsetzen des Blasenstromes $(t \approx 650 \mathrm{~s})$. Die Anwesenheit von Blasen senkt die mittlere relative Permittivität der Flüssigkeit zwischen den Elektroden 1 und 3 und lässt $C_{13}$ abfallen. Bei Vernachlässigung dieses Effekts würde man den LN2-Füllstand unterschätzen. Die Teilkapazität $C_{25}$ hingegen wird nicht durch den Blasenstrom beeinflusst, da nur wenige elektrische Feldlinien zwischen Elektrode 2 und 5 durch den Blasenstrom verlaufen.

Wie zuvor begründet, sollte $C_{13}(L)$ verwendet werden, um $L$ im Bereich von 0 bis $20 \mathrm{~mm}$ zu schätzen, während $C_{25}(L)$ sich besser für $L$ zwischen 15 und $30 \mathrm{~mm}$ eignet. Wie Abb. 9 zeigt, steigt das Rauschen im Messergebnis für $L$ stark an, sobald diese Kriterien missachtet werden.

Das in Abb. 9 einmontierte Foto ist ein Standbild des Videos, das zu dem Moment gehört, in dem der LN2-Füllstand $L$ aufgrund des Blasenstroms abfällt. Aufgrund der Blasen „kocht“ die Oberfläche der Flüssigkeit.

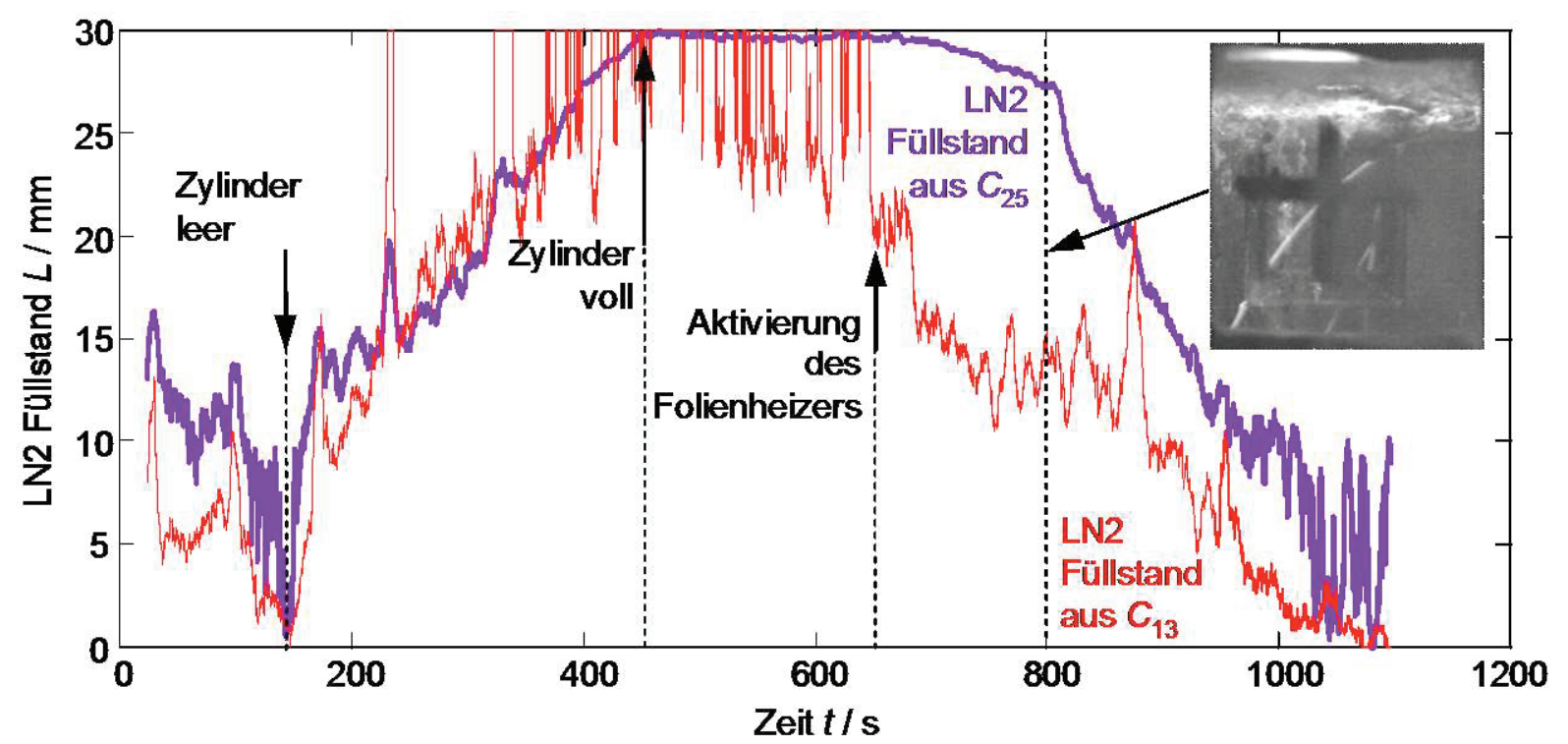

Abb. 9: Flüssigkeitsfüllstand im Zylinder, einmal gemessen über das Elektrodenpaar 1-3 (rote Kurve) und einmal gemessen über das Paar 2-5 (violette Kurve). 

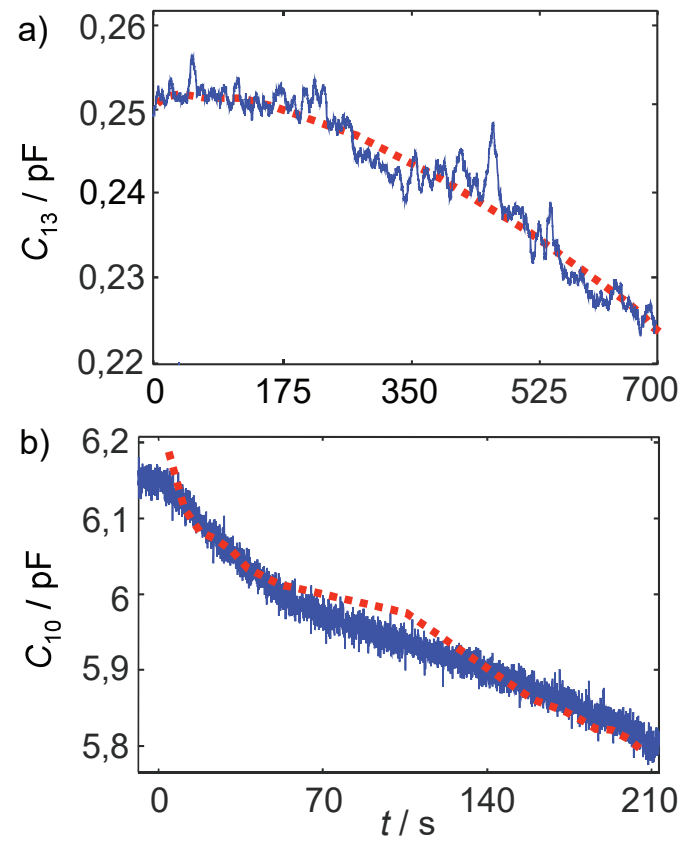

Abb. 10: Gemessene (blau durchgezogen) und per FE simulierte (rot gepunktet) Kapazitäten bei Veränderung des Gasvolumens im Zylinderinnenraum. a) Teilkapazität $\mathrm{C}_{13}$ zwischen den Elektroden 1 und 3 (vgl. Abb. 7) während einer Zunahme des Gasvolumens (Abnahme des LN2-Volumens). b) Kapazität gegen Masse $\mathrm{C}_{10}$ (siehe $A b b$. 6a) während einer Zunahme des Gasvolumens (Abnahme des LN2-Volumens).

Der Rechnungs-Messungs-Vergleich fällt hervorragend aus, wie Abb. 10 anhand zweier Fälle demonstriert. Dabei wurde für die simulierte Kapazität $C_{13}$ dieselbe Nichtidealität (Massepotentialabweichung) verwendet, die der Abb. 7 zugrunde lag.

Sowohl die gemessenen Teilkapazitäten $\left(C_{13}\right.$ in Abb. 10a) als auch die gemessenen Kapazitäten gegen Masse $\left(C_{10}\right.$ in Abb. 10b) stimmen ausgezeichnet mit den Modellvorhersagen überein. Wir ziehen folgende Schlüsse aus den Ergebnissen:

- Die Messergebnisse können qualitativ und quantitativ richtig mit Hilfe numerischer Berechnungen (nämlich von Kapazitäten) gedeutet werden.

- Bei der praktischen Kapazitätsmessung treten nichtideale Massepotentiale auf, die eine Rolle spielen und die bei der Interpretation der Messwerte zu berücksichtigen sind. Dies gelingt durch den hier verfolgten modellbasierten Ansatz.

- Die Messkurven sind verrauscht und besitzen einen für jede Elektrodenkombination charakteristischen additiven Offset, welcher durch Zuleitungskapazitäten hervorgerufen wird und von den Rohdaten subtrahiert werden muss. Zudem müssen die Kurven unter Umständen geglättet werden. Hier wurde z. B. die Kurve aus Abb. 10a vor der Darstellung mit einem gleitenden Mittelwertbilder der Breite 200 geglättet. Die Abtastrate betrug $25 \mathrm{~Hz}$, so dass 200 Messpunkte einer Zeitdauer von $8 \mathrm{~s}$ entsprechen.

- Die Erkennung von Kapazitätsänderungen im Bereich von wenigen fF stellt kein Problem für das realisierte Messsystem dar.

\section{Fazit und Ausblick}

Es wurde demonstriert, dass Phasenänderungen in flüssigem Stickstoff durch die Messung der elektrischen Kapazität zwischen verschiedenen Elektroden eines Mehrelektrodensystems an der Oberfläche eines Raumbereichs in situ erkannt werden können. Dies geschieht ohne erkennbaren Wärmeeintrag oder anderweitige Beeinträchtigungen des Mediums zwischen den Elektroden.

Abhängig von der Elektrodengeometrie lassen sich aus den gemessenen Teilkapazitäten und den Kapazitäten gegen Masse auch Phasenänderungen von Stoffen mit vergleichsweise geringen Unterschieden in der relativen Permittivität nachweisen.

Die Eigenschaften des Systems lassen sich durch entsprechende FE-Simulationen gut beschreiben. Auch stehen die Ergebnisse in guter Übereinstimmung mit Phasengrenzen, die bildgebend (mit einer Kamera) ermittelt wurden.

\section{Literaturnachweis}

[1] M. Siegl et al.: „Advanced Sensor technologies for cryogenic liquid propellant flow phenomena", Proc. 66th Int'l Astronautical Congr., Jerusalem, 12.-16. Okt. 2015.

[2] M. Mukhopadhyay, „Fundamentals of cryogenic engineering," PHI Learning, Neu Delhi, 2010.

[3] Span, R. et al.: „A Reference Equation of State for the Thermodynamic Properties of Nitrogen for Temperatures from 63.151 to $1000 \mathrm{~K}$ and Pressures to 2200 MPa", J. Phys. Chem. Ref. Data, Vol. 29, No. 6, S. 1361-1433, 2000.

[4] E. J. Murphy et al., „The Dielectric Properties of Insulating Materials", Bell Systems Technical Journal, Vol. 14, No. 4, S. 493-512, Okt. 1937.

[5] N. N., "PCap02A - Single-chip Solution for Capacitance Measurement" (Vers. 1.6), Datenblatt Acam messelectronic, 29. Mai 2014.

[6] S. Mühlbacher-Karrer, H. Zangl, „Light Weight Signal Processing for a Wireless Capacitive Sensing Platform for Mobile Applications", Proc. Sensor 2015, Nürnberg, S. 190-197, Mai 2015. 